\title{
Studying Bio-Inspired Coalition Formation of Robots for Detecting Intrusions Using Game Theory
}

\author{
Xiannuan Liang and Yang Xiao, Senior Member, IEEE
}

\begin{abstract}
In this paper, inspired by the society of animals, we study the coalition formation of robots for detecting intrusions using game theory. We consider coalition formation in a group of three robots that detect and capture intrusions in a closed curve loop. In our analytical model, individuals seek alliances if they think that their detect regions are too short to gain an intrusion capturing probability larger than their own. We assume that coalition seeking has an investment cost and that the formation of a coalition determines the outcomes of parities, with the detect length of a coalition simply being the sum of those of separate coalition members. We derive that, for any cost, always detecting alone is an evolutionarily stable strategy (ESS), and that, if the cost is below a threshold, always trying to form a coalition is an ESS (thus a three-way coalition arises).
\end{abstract}

Index Terms-Bio-inspired, coalition, game theory, intrusion detection, mobile sensors, robots.

\section{INTRODUCTION}

C OALITION is a term that indicates a union or an alliance in the society of animals. There are many instances of this phenomenon in nature, and there are many studies of coalition formation for animal studies [5], [8]-[14], [16], [17], [19], [20], [22], [24], [27]-[31], [33], [35], [39], [40]. However, there is no work on coalition formation of robots to detect intrusions using game theory. In this paper, which is inspired by the society of animals, we study coalition formation of robots to detect intrusion using game theory. We consider a group of robots (or mobile sensors) patrolling along a route to catch intrusion objects, such as tanks, human beings, etc., in a surveillance application. Robots move along the route with some speed. They have the capability to catch the intrusions, and their collaboration or coalition can enhance their ability to catch intrusions with some cost as a tradeoff. Our goal is to study the tradeoff of this collaboration. One theory that can be used to study collaboration or coalition is game theory, which (we believe) is a good tool to study the tradeoff of collaboration or coalition. Game theory has been used to model the collaboration and coalition of primates before, and we will introduce this in the next section.

We consider a group of robots of a certain size. If we assume that there is no difference in detection ability among

Manuscript received December 31, 2008; revised July 3, 2009. First published November 20, 2009; current version published June 16, 2010. This work was supported in part by the U.S. National Science Foundation (NSF) under Grant CCF-0829827, Grant CNS-0716211, and Grant CNS-0737325. This paper was recommended by Associate Editor T. Vasilakos.

The authors are with the Department of Computer Science, The University of Alabama, Tuscaloosa, AL 35487-0290 USA (e-mail: yishengyici2004@ yahoo.com; yangxiao@ieee.org).

Color versions of one or more of the figures in this paper are available online at http://ieeexplore.ieee.org.

Digital Object Identifier 10.1109/TSMCB.2009.2034976 the studied robots, then using more robots will improve the intrusion detection probability but will also increase the cost. Therefore, applying a small-sized group of robots is one of the practical ways to detect intrusions in routines. In this paper, to simplify the problem, we only consider three robots as a group. Intuitively, three robots are enough to insure a certainty of successfully capturing an intrusion since the intrusion in a 2-D plane can be well covered by the robots in three different directions. In our model, we assume that any robot tries to form a coalition with other robots when it believes that it is too weak to capture an intrusion alone. We consider an among-individual variation in detection strength, which is defined as the length of the detection region. We also take into account a potential investment cost of forming a coalition through estimation of the coalition. For the reasons that we will present in the next section for the analysis of coalitions in animal societies, we begin our work with triads of robots. Furthermore, like the work in [16] on the analysis of coalitions in animal society, we are interested in identifying conditions under which coalition forms and those under which always detecting intrusions separately pays.

The purpose of this paper is to also study collaborative robots via mathematical modeling. This modeling provides us with deep insights into the impacts of collaborations and can provide guides on how to collaborate in collaboration systems. This paper can be related to the following topics in collaboration: intelligent and autonomous agents (robots are treated as mobile agents) in collaboration, mobile and wireless collaboration systems (robots are treated as mobile sensors), multiagent systems in collaboration, and modeling of collaboration.

The rest of this paper is organized as follows: Section II provides some related work on animal coalitions and an introduction of evolutionarily stable strategy (ESS). Section III presents our math model. Sections IV and V give the detection probabilities of a single robot in the loop and robot coalition in the loop, respectively. Section VI presents our robot coalition game for detecting intrusions. We provide some evaluations in Section VII and finally conclude this paper in Section VIII.

\section{RELATED WORK AND BACKGROUND}

\section{A. Survey of Animal Coalition}

Coalition is a term to indicate a union or alliance in the society of animals. There are many instances for this phenomenon in nature [5], [8]-[14], [16], [17], [19], [20], [22], [24], [27][31], [33], [35], [39], [40]. Table I shows a short survey of animal coalitions.

Several quantitative models of coalition formation in natural systems have appeared in the related literature [2], [4], [6], [7], 
TABLE I

SuRvey OF ANIMAL COALITIONS

\begin{tabular}{|l|l}
\hline \multicolumn{1}{|c|}{ Papers } & \multicolumn{1}{c}{ Contents } \\
\hline Caro [11] & Collective mobbing of predators \\
\hline $\begin{array}{l}\text { Zabel } \text { et al. } \\
{[17]}\end{array}$ & $\begin{array}{l}\text { forming groups of conspecifics to fight for a higher } \\
\text { dominance rank }\end{array}$ \\
\hline $\begin{array}{l}\text { Parker } \text { et al. } \text { [5] } \\
\text { and Caro [13] }\end{array}$ & male coalitions for detecting and gaining females \\
\hline$[19],[33]$ & $\begin{array}{l}\text { gaining females: Western Australia, male bottlenose } \\
\text { dolphins forming alliance of 2 or more to herd, escort, } \\
\text { and defend them }\end{array}$ \\
\hline$[3],[9]$ & $\begin{array}{l}\text { gaining females: two or more subordinates of male } \\
\text { avanna baboons may try to beat a more dominant one } \\
\text { in consort of an estrous female, by forming a union of } \\
\text { two or more. }\end{array}$ \\
\hline
\end{tabular}

TABLE II

Quantitative Models of Animal Coalitions

\begin{tabular}{|l|l|}
\hline \multicolumn{1}{|c|}{ Papers } & \multicolumn{1}{c|}{ Contents } \\
\hline Noe [6] & $\begin{array}{l}\text { Presenting a simple "veto game" model for the } \\
\text { allocation of the payoff of coalitions in the } \\
\text { baboon societies }\end{array}$ \\
\hline Noe [2] & $\begin{array}{l}\text { Evaluating the possible fighting powers of the } \\
\text { dyadic coalition by summing up the powers of } \\
\text { both the members in each coalition, and arguing } \\
\text { that medium-strength individuals with each other } \\
\text { most frequently form collations. }\end{array}$ \\
\hline $\begin{array}{l}\text { Dugatkin [4] and } \\
\text { Johnstone [18] }\end{array}$ & $\begin{array}{l}\text { Studying the conditions under which individuals } \\
\text { considered may have motivation to join in fights } \\
\text { on the premise that helping one of the conspecfic } \\
\text { beat its opponent increasing the probability to } \\
\text { beat the looser subsequently }\end{array}$ \\
\hline $\begin{array}{l}\text { Pandit and van } \\
\text { Schaik [1] }\end{array}$ & $\begin{array}{l}\text { Providing a model for primates in which the } \\
\text { coalition of the challengers challenge an } \\
\text { individual ranked higher, and arguing that the } \\
\text { formation of challengers' combination most } \\
\text { likely occurs when despotism has a low } \\
\text { likelihood to come out in their environment. }\end{array}$ \\
\hline $\begin{array}{l}\text { Whitehead and } \\
\text { Connor [7] }\end{array}$ & $\begin{array}{l}\text { Adopting analytical study and simulation models } \\
\text { to explore the alliance size and the influence on } \\
\text { their propensity to form coalitions when the } \\
\text { encountering rate of the individuals varies. }\end{array}$ \\
\hline $\begin{array}{l}\text { Mesterton-Gibbons } \\
\text { [16] }\end{array}$ & $\begin{array}{l}\text { Assuming that there is a potential investment } \\
\text { cost of forming a coalition and that any of the } \\
\text { potential collaborators does not know the } \\
\text { strengths of others but only its own strength; } \\
\text { allowing that the fighting abilities among } \\
\text { individuals vary rather than keep fixed; and as } \\
\text { the first attempt, examining the effect to the } \\
\text { collation structure when the cost of fights varies } \\
\text { and analytically indentifying evolutionarily } \\
\text { stable strategies (ESSs) regarding coalition } \\
\text { formation }\end{array}$ \\
\hline
\end{tabular}

[16], [18], [38]. Table II provides a short survey of quantitative models of animal coalitions.

In related literature (e.g., [1], [15], and [32]), it is shown that, in general, primates form coalitions in an average size of slightly more than 2 . Therefore, coalitions can be assumed to consist of two or three individuals. The authors in [16], [18], and [25] consider triadic interactions to be so important in the study of coalitions because triads are groups that can most practically be studied and analyzed, particularly in the case that variation in fighting strengths among individuals is taken into consideration. Furthermore, Mesterton-Gibbons and Sherratt [16] examine and analyze coalition formations in triads. In the analysis, they focus on finding out the conditions under which a true coalition (two versus one) has the greatest probability of arising. Note that a "true coalition" usually does not include the "grand coalition" that is formed by all the individuals in game theory [16] or behavioral ecology [26].

A survey of primate (monkey) social systems, scent marking, and their applications in mobile and static sensor networks is provided in [46]. Primate-inspired communication mechanisms are proposed and studied in [43]. A bio-inspired scent-marking mechanism is proposed and studied in [44]. A survey of bio-inspired attentions is given in [47]. Collaborations among robots, sensors, and radio-frequency identification tags are studied in [42], [45], and [48].

However, there is no work on coalition formation of robots to detect intrusions using game theory. In this paper, which is inspired by the society of animals, we study coalition formation of robots to detect intrusion using game theory. A short version (i.e., the preliminary work) of this paper was presented in [41].

\section{B. Game Theory: ESS}

ESS [23] is a concept in game theory. An ESS is a refined form of a Nash equilibrium, which is also a concept in game theory. The definition of one Nash equilibrium (in the case of a dyadic game) is as follows: A strategy pair is called a Nash equilibrium when it satisfies the following condition: once the players in the game have chosen their strategies, each of the players can gain more benefit by keeping its current strategy than by replacing its choice with any alternative strategy under the condition that at each time there is only one potential mutant that may change its choice of strategy. Let $\mathrm{P}(u, v)$ represent the payoff of a player holding strategy $u$ against another one holding strategy $v$. In other words, $\mathrm{P}(u, v)$ represents the first player's payoff. A larger payoff means more benefits. The strategy pair $(u, u)$ is considered to be one Nash equilibrium when and only when the following condition is satisfied: $\mathrm{P}(u, u) \geq$ $\mathrm{P}(v, u)$ for all $v \neq u$. This definition of Nash equilibrium takes into consideration the possibility that, to any of the players, there exist a strategy $v$ that is different with $u$ but is as good as $u$. Under the assumption that there is no long-term motivation for players to choose $v$ instead of $u$, which is as good as $v$, Nash equilibrium is supposed to be stable. This fact gives birth to the derivation of the definition of the ESS. Smith and Price [36] define an ESS as follows: An ESS is a strategy $u$ that satisfies, for all $v \neq u$, either 1) $\mathrm{P}(u, u)>\mathrm{P}(v, u)$, or 2) $\mathrm{P}(u, u)=\mathrm{P}(v, u)$ and $\mathrm{P}(u, v)>\mathrm{P}(v, v)$. In other words, an ESS satisfies either the first condition or the second condition. The first condition is also called strict Nash equilibrium. The second condition indicates that although changing strategy $u$ to strategy $v$ is neutral, the population of players choosing strategy $u$ has an advantage compared to choosing $v$.

In most simple games, the ESSs and Nash equilibriums imply each other. For example, in the famous case in game theory called Prisoner's Dilemma [23], the police separate two suspects without enough evidence and lure one to testify against another. As shown in Table III, if both stays silent 
TABLE III

PRISONER's DILEMMA [23]

\begin{tabular}{|l|l|l|}
\hline & Cooperating & Defecting \\
\hline Cooperating & $-2,-2$ & $-7,-1$ \\
\hline Defecting & $-1,-7$ & $-4,-4$ \\
\hline
\end{tabular}

TABLE IV

HARM THY NEIGHBOR [23]

\begin{tabular}{|l|l|l|}
\hline & $u$ & $v$ \\
\hline$u$ & 3,3 & 2,3 \\
\hline$v$ & 3,2 & 3,3 \\
\hline
\end{tabular}

(i.e., collaborating each other), then both of them will be sentenced to two months. If they betray (i.e., defect) each other, then both of them will be sentenced to four months. If one stays silent, but the other does not, then the one being silent will be sentenced to seven months, and the other will be sentenced to one month. Note that the payoff is defined as the negative value of the months sentenced. It is clearly that in the Prisoner's Dilemma [23], as shown in Table III, (Defecting, Defecting) is an Nash equilibrium, and Defecting is an ESS since $\mathrm{P}($ defecting, defecting $)=-4>\mathrm{P}($ cooperating, defecting $)=-7$.

In some games, Nash equilibriums may not be equivalent to ESSs. For instance, in Harm Thy Neighbor [23] (shown in Table IV), two Nash equilibriums $(u, u)$ and $(v, v)$ exist because, in each of the strategy pairs, each of the players cannot benefit more by choosing a new strategy. Nevertheless, only one ESS exists, which is not strategy $u$ but strategy $v$, since strategy $u$ does not satisfy all the conditions to be an ESS. Let us assume that strategy $u$ is an ESS, and then we derive contradiction as follows. Based on the definition of ESS, since we have $\mathrm{P}(v, u)=\mathrm{P}(u, u)=3$, i.e., the first condition of the definition of ESS does not hold, then the second condition must hold, i.e., $\mathrm{P}(u, v)>\mathrm{P}(v, v)$. However, based on Table IV, $\mathrm{P}(u, v)=2<$ $\mathrm{P}(v, v)=3$, and this is contradicted. This proves that strategy $u$ is not an ESS. Since we have $\mathrm{P}(v, v)=3>\mathrm{P}(u, v)=2$, the first condition of the definition of ESS holds. Therefore, strategy $v$ is ESS.

\section{Math Model for Robot CoAlition}

We are studying the coalition formation of a group of three robots $(\mathrm{A}, \mathrm{B}$, and $\mathrm{C})$ that are detecting and trying to capture intrusions in a closed loop with a length $3 L$, as shown in Fig. 1. Note that in this paper, when we describe an arc in a loop, we always use anti-clock direction. The intrusions occur one by one in the loop. Once an intrusion occurs, it keeps still for a time period and then disappears. All of the robots have a velocity of $V$. Let $D(X)$ denote the detection region (length) of robot $X$. The detect regions (lengths) of robots $\mathrm{A}, \mathrm{B}$, and $\mathrm{C}$ are $D(A)$, $D(B)$, and $D(C)$, respectively. $D(A), D(B)$, and $D(C)$ are random variables with the same uniform probability density function as

$$
g(x)= \begin{cases}1 / L, & 0<x \leq L \\ 0, & \text { otherwise }\end{cases}
$$

The intrusion capturing probability of a party (i.e., a robot or robot coalition) is defined as the intrusion first detection prob- ability of the party times the conditional capturing probability of the party. The intrusion capturing probability of a group is equal to the summation of the intrusion capturing probabilities of all the parties in the group.

We have the following assumptions for our model.

1) An intrusion can be detected if it is in the detect region (length) of a robot.

2) An intrusion can only be captured by the robot or robot coalition (called "a party") that detects it first.

3) Intrusions independently occur one by one.

4) Each intrusion uniformly occurs in the loop, i.e., each intrusion appears with a probability density $1 /(3 L)$ at any point of the loop.

5) After an intrusion occurs on the loop, it will last a time period. The lasting time of each intrusion is subject to an exponential distribution with a mean $1 / \mu$, i.e.,

$$
f(x)= \begin{cases}\mu e^{-\mu x}, & 0<x<+\infty \\ 0, & \text { otherwise }\end{cases}
$$

6) There are time periods in which there is no intrusion on the loop. Each no intrusion time period is subject to an exponential distribution with a mean $1 / \lambda$.

7) A robot will try to form a coalition with other robots if its detect length is too low. The cost (called "investment cost") of the robot who seeks a coalition is $\theta \Lambda$, where the group fitness is $\Lambda$, and we have $0<\theta<+\infty$.

8) Under the condition of being first detected, the intrusion capturing probability is as follows: (Case 1) $1 / 3$ whichever robot detects it first if there are three separate robots and there is no coalition; (Case 2) 1 if there is a three-way coalition; (Case 3a ) 1/2 if there is a twoway coalition (or true coalition) and it is first detected by the coalition; and (Case 3b) 1/3 if there is a two-way coalition (or true coalition) and it is first detected by the other robot.

9) Under the notations defined in Table V, assume that the robots are deployed as follows. First, if there is no coalition, as shown in Fig. 1(a), we have $\operatorname{arc}(\mathrm{c} 2, \mathrm{a} 1)=\operatorname{arc}(\mathrm{b} 2, \mathrm{c} 1)=\operatorname{arc}(\mathrm{a} 2, \mathrm{~b} 1)=Q / 3$, where $Q=3 L-D(A)-D(B)-D(C)$. Second, if it is a two-way coalition $A C$ versus one single robot $B$, as shown in Fig. 1(b), $A C$ is the two-way coalition with no overlapping between their detection lengths, and we have $\operatorname{arc}(\mathrm{a} 2, \mathrm{~b} 1)=\operatorname{arc}(\mathrm{b} 2, \mathrm{c} 1)=Q / 2$. This rule is also applied to other cases of two-way coalition $(X Y)$ versus one single robot $Z$ when $X Y \neq A C$. Third, the total detect length of a coalition is the sum of the detect lengths of the members of the coalition, i.e., there is no overlapping between the detect regions of any pair of robots, as shown in Fig. 1.

10) (Assumptions about the fitness) The probability that an intrusion can be captured is 1 in the case that all three robots have a detect length $L$ and form a three-way coalition. In other cases, the probability is less than or equal to 1 . We use the maximum capturing probability to scale the group fitness and let it equal to 1 , i.e., $\Lambda=1$. 


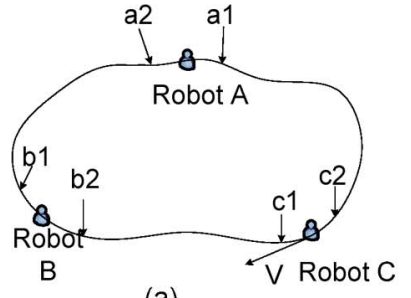

(a)

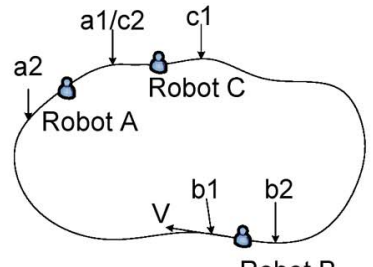

(b)

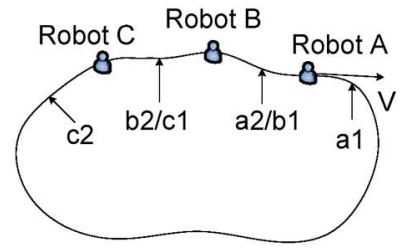

(c)

Fig. 1. Three cases of coalition formation [the length of the closed loop is $3 L$, and the speed of each robot is $V$; the detect lengths of robots A, B, and C are $\operatorname{arc}(\mathrm{a} 1, \mathrm{a} 2), \operatorname{arc}(\mathrm{b} 1, \mathrm{~b} 2)$, and $\operatorname{arc}(\mathrm{c} 1, \mathrm{c} 2)$, respectively; parties (a party is a robot or coalition) are separated by an equal distance]. (a) No coalition. (b) Two-way coalition versus one single robot. (c) Three-way coalition.

11) (Assumptions for the allocation of the rewards) As we will know that, to any intrusion, when a certain party has the same capturing probability, we consider the intrusion capturing probability to be the reward of the party. Therefore, the allocation of the reward within the party depends on the following different cases: (Case 1) Each robot has its own capturing probability as its reward if there is no coalition. (Case 2) Each robot in the party (three-way coalition) has $1 / 3$ of the capturing probability of the party as its reward if it is a three-way coalition. (Case 3) Each robot in the party of the coalition has $1 / 2$ of the capturing probability of the party if it is two versus one (true coalition), and the other has its own capturing probability as its reward.

Based on the assumptions and the math model, we will work on the following two problems:

- Problem 1: What is the average payoff for a potential mutant with strategy $w$ when the population has strategy $y$ ?

- Problem 2: Does an ESS exist? If there is any, then under what conditions does it exist?

Notations and some definitions in this paper are listed in Table V.

Based on the preceding assumptions and notations, we have

$$
\begin{aligned}
D(X Y) & =D(Y X)=D(X)+D(Y) \\
D(A B C) & =D(A)+D(B)+D(C) .
\end{aligned}
$$

To solve Problem 1, we first introduce a definition, e.g., first the detection probability of a party $S$, which we denote as $P_{d}(S)$. We know that, for one certain intrusion, there is a probability that it is first detected by $S$.

\section{Detection Probability of Single Robot}

We first work on a robot with the same parameters as our model but with just one robot $X$, as shown in Fig. 1(a), but only one robot $X$ in the loop. We have the following theorem.

Theorem 1: Assuming that there is only one robot $X$ with the detection length $D(X)$, according to the foregoing notations and assumptions, we have

$$
P_{d}(X)=\frac{D(X)}{3 L}+\frac{V}{3 L \mu}-\frac{V}{3 L \mu} e^{-\frac{\mu(3 L-D(X))}{V}} .
$$

Proof: An intrusion could be detected in two situations: one is observed by robot $X$ when it happens, i.e., the intrusion happens in the detection range of the robot when it appears and

\begin{tabular}{|c|c|}
\hline $\mathrm{A}, \mathrm{B}$, and $\mathrm{C}$ & Three robots \\
\hline $\mathrm{X}, \mathrm{Y}$, or Z & $\begin{array}{l}\text { Any robot among } \mathrm{A}, \mathrm{B} \text {, and } \mathrm{C} ; \mathrm{X}, \mathrm{Y} \text {, and } \mathrm{Z} \text { are } \\
\text { diffident }\end{array}$ \\
\hline $\mathrm{D}(\mathrm{X})$ & Detection length of robot X \\
\hline $\mathrm{XY}=\mathrm{YX}$ & $\begin{array}{l}\text { A coalition of robots } \mathrm{X} \text { and } \mathrm{Y} \text {. We have } \mathrm{XY}=\mathrm{YX} \\
\text { and } X Y \in\{A B, A C, B C\} \text {. }\end{array}$ \\
\hline $\mathrm{ABC}$ & A coalition of robots $A, B$, and $C$. \\
\hline $3 L$ & Length of the closed loop \\
\hline$P_{d}(X)$ & $\begin{array}{l}\text { The probability that any certain intrusion is first } \\
\text { detected by the robot } X\end{array}$ \\
\hline$P_{d}(X Y)$ & $\begin{array}{l}\text { The probability that any certain intrusion is first } \\
\text { detected by the coalition } X Y\end{array}$ \\
\hline$P_{d}(A B C)$ & $\begin{array}{l}\text { The probability that any certain intrusion is first } \\
\text { detected by the coalition } A B C\end{array}$ \\
\hline $\mathrm{P}(u, v)$ & $\begin{array}{l}\text { The payoff of the player who holds a strategy } u \\
\text { when the other player holds a strategy } v \text { in the } \\
\text { two-player games. }\end{array}$ \\
\hline$\Lambda$ & The group fitness, in this paper, $\Lambda=1$ \\
\hline$\theta$ & $\begin{array}{l}\text { The investment cost when a robot tries forming } \\
\text { a coalition with other robots }\end{array}$ \\
\hline $\mathrm{P}[$ loss $]$ & $\begin{array}{l}\text { The probability that an intrusion is not first } \\
\text { captured by a party }\end{array}$ \\
\hline $\mathrm{P}\left[\right.$ loss $\left.\mid t_{0}\right]$ & $\begin{array}{l}\text { The probability that an intrusion is not first } \\
\text { captured by a party under the condition that the } \\
\text { intrusion occurs at time } t_{0}\end{array}$ \\
\hline $\bar{y}$ & $\begin{array}{l}\text { The variable which presents the point at which } \\
\text { the first intruder occurs }\end{array}$ \\
\hline$V$ & The moving speed of the robots \\
\hline$w$ & The strategy of the potential mutant \\
\hline$y$ & The strategy of each of the population \\
\hline$f(w, y)$ & $\begin{array}{l}\text { The payoff the potential mutant that holds } \\
\text { strategy } w \text { when each of the population holds } \\
\text { strategy } y\end{array}$ \\
\hline $\mathrm{S}$ & A party. $S \in\{A, B, C, A B, A C, B C, A B C\}$ \\
\hline No coalition & $\forall$ any party $S, S \in\{A, B, C\}$ \\
\hline True coalition & $\begin{array}{l}\exists \text { a party } S, S \in\{A, B, C\}, \text { and the other party } \\
\in\{A B, A C, B C\}\end{array}$ \\
\hline two-way coalition & True coalition \\
\hline two versus one & True coalition \\
\hline three-way coalition & $\forall$ any party $S, S \in\{A B C\}$ \\
\hline potential mutant & The player that is likely to change its strategy \\
\hline population & The rest of the players in the triad \\
\hline$Q$ & $\begin{array}{l}3 L-D(A)-D(B)-D(C) \\
=3 L-D(X)-D(Y)-D(Z)\end{array}$ \\
\hline$P_{o, i}(X)$ & $\begin{array}{l}\text { the payoff of the potential mutant } X \text { in case } i \text {, } \\
\text { where } i=1, \ldots, 8 \text {. }\end{array}$ \\
\hline$h(w, y, z)$ & $\begin{array}{l}\text { the average payoff in a more general form that } \\
\text { three individuals play strategies } w, y, z, \\
\text { respectively }\end{array}$ \\
\hline$\theta_{1}$ & $\begin{array}{l}\text { threshold of } \theta \text {, below which strategy } L \text { is an } \\
\text { ESS. }\end{array}$ \\
\hline
\end{tabular}

TABLE $\mathrm{V}$

NOTATIONS AND SOME DEFINITIONS

thus is immediately detected, and the other situation is one in which the intrusion is not observed by the robot at the beginning but is still detected before it disappears. 
What we are really interested in is the second situation, in which we want to know if the intrusion can be detected if it does not happen in the robot's coverage at the beginning.

To calculate the probability that an intrusion will be detected (also first detected since there is only one robot in the loop) by robot $X$, let $\mathrm{P}[$ loss $]$ denote the probability that an intrusion will not first be detected by robot $X$. We know that the variable of the time duration from the beginning (called as time zero) to the instant when the first intrusion occurs has an exponential distribution function with a mean of $1 / \lambda$. Under the condition that the first intrusion occurs at instant $t_{0}$, there is a conditional probability that the first intruder is not detected. We denote it as $\mathrm{P}\left[\operatorname{loss} \mid t_{0}\right]$. To calculate $\mathrm{P}\left[\operatorname{loss} \mid t_{0}\right]$, let us first calculate the conditional probability that the first intruder is not detected under the condition that the first intrusion occurs in a certain point $\bar{y}$ in the closed loop at the instant $t_{0}$. Let $\|\bar{y}\|$ denote the distance between point $\bar{y}$ and the detection range of robot $X$ at the instant $t_{0}$. We denote the conditional probability as $\mathrm{P}\left[\operatorname{loss} \mid\|\bar{y}\|=s, t_{0}\right]$.

Obviously, if the first intrusion occurs at the detect region of the robot at the instant $t_{0}$ (i.e., $\|\bar{y}\|=s=0$ ), then we have $\mathrm{P}\left[\operatorname{loss} \mid\|\bar{y}\|=s=0, t_{0}\right]=0$. Supposing that the first intrusion occurs at the position of point $\bar{y}$ of the closed loop in the undetected region of robot $X$, we have $\mathrm{P}\left[\operatorname{loss} \mid\|\bar{y}\|=s \neq 0, t_{0}\right] \neq 0$.

Under the condition that the first intrusion occurs in point $\bar{y}$ at the instant $t_{0}$, it is not captured if and only if its lasting time is shorter than the time duration from $t_{0}$ to the instant when the detect range of the robot begins to cover point $\bar{y}$. Then, the time duration from $t_{0}$ to the instant when the detect range of the robot begins to cover point $\bar{y}$ is $s / V$. Furthermore, we have

$$
P\left[\operatorname{loss} \mid\|\bar{y}\|=s, t_{0}\right]=\int_{0}^{s / V} \mu e^{-\mu t} d t=1-e^{-\mu s / V} .
$$

From the assumptions, since each intrusion appears in any point of the loop with a probability density $1 /(3 L)$, we can easily obtain

$$
\begin{aligned}
\mathrm{P}\left[\operatorname{loss} \mid t_{0}\right] & =\int_{0}^{3 L-D(X)} \mathrm{P}\left[\operatorname{loss} \mid\|\bar{y}\|=s, t_{0}\right] \frac{1}{3 L} d s \\
& =\int_{0}^{3 L-D(X)}\left(1-e^{-\mu \frac{s}{V}}\right) \frac{1}{3 L} d s \\
& =1-\frac{D(X)}{3 L}-\frac{V}{3 L \mu}+\frac{V}{3 L \mu} e^{-\frac{\mu(3 L-D(X))}{V}} .
\end{aligned}
$$

Obviously, $\mathrm{P}\left[\operatorname{loss} \mid t_{0}\right]$ is constant when $t_{0}$ varies. Therefore, we have $\mathrm{P}[\operatorname{loss}]=\mathrm{P}\left[\operatorname{loss} \mid t_{0}\right]$. This is also the memoryless property. We can easily extend the conclusion about the probability that the first intruder is not being captured to the case of the $i$ th intruder. Similar to the deduction of the case of the first intruder, we can obtain

$$
\begin{aligned}
& \mathrm{P} \text { [the } i \text { th intruder is not captured }] \\
& \quad=\mathrm{P}[\mathrm{loss}] \\
& \quad=1-\frac{D(X)}{3 L}-\frac{V}{3 L \mu}+\frac{V}{3 L \mu} e^{-\frac{\mu(3 L-D(X))}{V}} .
\end{aligned}
$$

From the preceding derivation, we know that each intrusion has the same probability of not being detected. That signifies that each intrusion has the same probability of being detected (also first detected). Therefore, we have $P_{d}(X)=1-\mathrm{P}[\mathrm{loss}]$. This concludes the proof of Theorem 1 .

From Theorem 1, we know that, with any pair of independent intrusion events $E_{1}$ and $E_{2}$, the probability that event $E_{1}$ is first detected by robot $X$ is the same as the one that $E_{2}$ is first detected by robot $X$. Therefore, in our model, $P_{d}(X)$ is the probability that robot $X$ first detects any certain intrusion.

\section{Detection Probabilities of Robot Coalition}

From Theorem 1, we can get $P_{d}(S)$ in following three cases, where $S$ is any party.

\section{A. Case 1: No Coalition}

Case 1 is for no coalition [shown in Fig. 1(a)]. We derive $P_{d}(X)$, and $X$ is any one robot among robots $A, B$, and $C$.

Let $Q=3 L-D(A)-D(B)-D(C)$. We have the following theorem.

Theorem 2: Assuming that there is no coalition among the three robots, for any robot $X$ among the three with the detection length $D(X)$, we have

$$
P_{d}(X)=\frac{D(X)}{3 L}+\frac{V}{3 L \mu}-\frac{V}{3 L \mu} e^{-\frac{\mu Q}{3 V}} .
$$

Proof: Note that the proof is similar to the proof of Theorem 1 . Therefore, we only describe those that are different.

Similar to the proof of Theorem 1, we have the corresponding definitions of $\mathrm{P}[\operatorname{loss}], t_{0}, \mathrm{P}\left[\operatorname{loss} \mid t_{0}\right]$, and $\bar{y}$. If $Q / 3<\|\bar{y}\|<$ $3 L-D(X)$, then the intrusion cannot first be detected by robot $X$ since the other two robots can detect it earlier than robot $X$. That is to say that $\mathrm{P}[\operatorname{loss} \mid Q / 3<\|\bar{y}\|=s<3 L-$ $\left.D(X), t_{0}\right]=1$. Furthermore, we have $0<\mathrm{P}[\operatorname{loss} \mid 0<\|\bar{y}\|=$ $\left.s<Q / 3, t_{0}\right]<1$. Similar to the proof of Theorem 1, we have

$$
\begin{aligned}
& P\left[\operatorname{loss} \mid 0<\|\bar{y}\|=s<Q / 3, t_{0}\right] \\
& \quad=\int_{0}^{s / V} \mu e^{-\mu t} d t=1-e^{-\mu s / V} \\
& \mathrm{P}\left[\operatorname{loss} \mid t_{0}\right] \\
& \quad=\int_{0}^{Q / 3} \mathrm{P}\left[\operatorname{loss} \mid\|\bar{y}\|=s, t_{0}\right] \frac{1}{3 L} d s+\int_{Q / 3}^{3 L-D(X)} 1 \frac{1}{3 L} d s \\
& \quad=\int_{0}^{Q / 3}\left(1-e^{-\mu \frac{s}{V}}\right) \frac{1}{3 L} d s+\frac{3 L-D(X)-Q / 3}{3 L} \\
& \quad=\frac{3 L-D(X)}{3 L}-\frac{V}{3 L \mu}+\frac{V}{3 L \mu} e^{-\frac{\mu Q}{3 V}} \\
& P_{d}(X)=1-\mathrm{P}[\operatorname{loss}]=1-\mathrm{P}\left[\operatorname{loss} \mid t_{0}\right] .
\end{aligned}
$$

This concludes the proof of Theorem 2 . 


\section{B. Case 2: Three-Way Coalition}

Three-way coalition can be considered to be a single robot with a detect length $D(A)+D(B)+D(C)$. Similar to Theorem 1, we can easily prove the following theorem.

Theorem 3: Assuming that there is a three-way coalition among the three robots, we have

$$
P_{d}(A B C)=\frac{D(A)+D(B)+D(C)}{3 L}+\frac{V}{3 L \mu}-\frac{V}{3 L \mu} e^{-\frac{\mu Q}{V}} .
$$

\section{Case 3: True Coalition}

We have the following theorem.

Theorem 4: Assuming that there is a two-way coalition versus one robot, in which robot $X$ and robot $Y \neq X$ form a true coalition, while robot $Z(Z \neq X, Z \neq Y)$ is the singe robot, we have

$$
\begin{aligned}
P_{d}(\mathrm{XY}) & =\frac{D(X)+D(Y)}{3 L}+\frac{V}{3 L \mu}-\frac{V}{3 L \mu} e^{-\frac{\mu Q}{2 V}} \\
P_{d}(\mathrm{Z}) & =\frac{D(Z)}{3 L}+\frac{V}{3 L \mu}-\frac{V}{3 L \mu} e^{-\frac{\mu Q}{2 V}} .
\end{aligned}
$$

Proof: Similar to the proof of Theorem 2, we can easily prove (5). For proving (4), similar to the proof of Theorem 2, we have

$$
\begin{aligned}
& \mathrm{P}\left[\operatorname{loss} \mid Q / 2<\|\bar{y}\|=s<3 L-D(X)-D(Y), t_{0}\right]=1 \\
& P\left[\operatorname{loss} \mid 0<\|\bar{y}\|=s<Q / 2, t_{0}\right]=\int_{0}^{s / V} \mu e^{-\mu t} d t=1-e^{-\mu s / V} \\
& \mathrm{P}\left[\operatorname{loss} \mid t_{0}\right]=\int_{0}^{Q / 2} \mathrm{P}\left[\operatorname{loss} \mid\|\bar{y}\|=s, t_{0}\right] \frac{1}{3 L} d s \\
& +\int_{Q / 2}^{3 L-D(X)-D(Y)} 1 \frac{1}{3 L} d s \\
& =\int_{0}^{Q / 2}\left(1-e^{-\mu \frac{s}{V}}\right) \frac{1}{3 L} d s+\frac{3 L-D(X)-D(Y)-Q / 2}{3 L} \\
& =\frac{3 L-D(X)-D(Y)}{3 L}-\frac{V}{3 L \mu}+\frac{V}{3 L \mu} e^{-\frac{\mu Q}{2 V}} \\
& P_{d}(X Y)=1-\mathrm{P}[\operatorname{loss}]=1-\mathrm{P}\left[\operatorname{loss} \mid t_{0}\right] .
\end{aligned}
$$

This concludes the proof of Theorem 4.

\section{Robot Coalition Game}

An ESS is a strategy that each of the players holds. Therefore, to judge whether a strategy $y$ is an ESS or not, we can consider the case that at the beginning each of the players holds the strategy $y$. Then, we examine whether any of the players can benefit more by shifting its strategy $y$ to another strategy $w$ when the rest of the players still hold strategy $y$. Thus, we can focus on one player and let its strategy vary. We call this player the potential mutant and the rest of the players the population. Note that in this paper the strategy is the alliance threshold.
As listed before, to any intrusion, when a certain party has the same capturing probability, we consider the intrusion capturing probability to be the reward of the party. Therefore, the allocation of the reward within the party depends on the following different cases: (Case 1) Each robot has its own capturing probability as its reward if there is no coalition. (Case 2) Each robot in the party (three-way coalition) has $1 / 3$ of the capturing probability of the party as its reward if it is a three-way coalition. (Case 3) Each robot in the party of the coalition has $1 / 2$ of the capturing probability of the party if it is two versus one (true coalition) and the other one has its own capturing probability as its reward.

As listed before, we assume that in the condition of being first detected, the intrusion capturing probability is as follows: (Case 1) 1/3 whichever robot detects it first if there are three separate robots and there is no coalition; (Case 2) 1 if there is a three-way coalition; (Case 3a) $1 / 2$ if there is a two-way coalition (or true coalition) and it is first detected by the coalition; and (Case $3 b$ ) $1 / 3$ if there is a two-way coalition (or true coalition) and it is first detected by the other robot.

When defining a payoff, we consider several aspects, such as intrusion capturing probability, a coalition or not, the shared payoff if it is a coalition, and the cost if it is a coalition.

\section{A. Solution of Problem 1}

Let $w(w \in[0, L])$ be the alliance threshold (i.e., a strategy) of potential mutant $X$, where $X$ is any one among $A, B$, and $C$. Let $y$ be the alliance threshold (i.e., strategy) of the population (robot $Y$ and robot $Z$ ). We have $D(X)+D(Y)+D(Z)=$ $D(A)+D(B)+D(C)$.

Eight mutually exclusive cases constitute the sample space of combination of the detect lengths $(D(X), D(Y)$, and $D(Z))$ as follows. Let $P_{o, i}(X), i=1, \ldots, 8$, denote the payoff of the potential mutant $X$ in case $i$.

Case 1: When $D(X)<w, D(Y)<y, D(Z)<y$, all of the three robots seek coalition so that, based on Theorem 3, we define

$$
\begin{aligned}
P_{o, 1}(X) & =\left(P_{d}(A B C)\right) / 3-\theta(\text { in Theorem } 3) \\
& =\frac{D(X)+D(Y)+D(Z)}{9 L}+\frac{V}{9 L \mu}-\frac{V}{9 L \mu} e^{-\frac{\mu Q}{V}}-\theta .
\end{aligned}
$$

In the preceding derivations, $P_{d}(A B C)$ is divided by 1 and then by 3 since 1 ) the conditional intrusion capturing probability is 1 if there is a three-way coalition, and 2) the reward should be shared by the three robots.

Case 2: When $D(X)<w, D(Y) \geq y, D(Z)<y$, robots $X$ and $Z$ seek coalition, but robot $Y$ does not, so based on Theorem 4 we define

$$
\begin{aligned}
P_{o, 2}(X) & =\left(P_{d}(\mathrm{XZ}) / 2\right) / 2-\theta(\text { in Theorem } 4) \\
& =\frac{D(X)+D(Z)}{12 L}+\frac{V}{12 L \mu}-\frac{V}{12 L \mu} e^{-\frac{\mu Q}{2 V}}-\theta .
\end{aligned}
$$


In the preceding derivations, $P_{d}(\mathrm{XZ})$ is divided by 2 for two times since 1) the conditional intrusion capturing probability is $1 / 2$ since there is a two-way coalition (or true coalition) and the intrusion is first detected by the coalition, and 2) the reward should be shared by robots $X$ and $Z$.

Case 3: When $D(X)<w, D(Y)<y, D(Z) \geq y$, robots $X$ and $Y$ seek coalition, but robot $Z$ does not, so based on Theorem 4 we define

$$
\begin{aligned}
P_{o, 3}(X) & =\left(P_{d}(\mathrm{XY}) / 2\right) / 2-\theta(\text { in Theorem } 4) \\
& =\frac{D(X)+D(Y)}{12 L}+\frac{V}{12 L \mu}-\frac{V}{12 L \mu} e^{-\frac{\mu Q}{2 V}}-\theta .
\end{aligned}
$$

Case 4: When $D(X) \geq w, D(Y)<y, D(Z)<y$, robots $Y$ and $Z$ seek coalition, but robot $X$ does not, so based on Theorem 4 we define

$$
\begin{aligned}
P_{o, 4}(X) & =\left(P_{d}(\mathrm{X}) / 3\right) / 1(\text { in Theorem } 4) \\
& =\frac{D(X)}{9 L}+\frac{V}{9 L \mu}-\frac{V}{9 L \mu} e^{-\frac{\mu Q}{2 V}} .
\end{aligned}
$$

Note that there is no $-\theta$ in (9).

Case 5: When $D(X)<w, D(Y) \geq y, D(Z) \geq y$, robot $X$ seeks coalition, but robots $Y$ and $Z$ do not, so based on Theorem 2 we define

$$
\begin{aligned}
P_{o, 5}(X) & =\left(P_{d}(\mathrm{X}) / 3\right) / 1-\theta(\text { in Theorem } 2) \\
& =\frac{D(X)}{9 L}+\frac{V}{9 L \mu}-\frac{V}{9 L \mu} e^{-\frac{\mu Q}{3 V}}-\theta .
\end{aligned}
$$

Case 6: When $D(X) \geq w, D(Y) \geq y, D(Z)<y$, all robots do not seek coalition, so based on Theorem 2 we define

$$
\begin{aligned}
P_{o, 6}(X) & =\left(P_{d}(\mathrm{X}) / 3\right) / 1(\text { in Theorem 2) } \\
& =\frac{D(X)}{9 L}+\frac{V}{9 L \mu}-\frac{V}{9 L \mu} e^{-\frac{\mu Q}{3 V}} .
\end{aligned}
$$

Case 7: When $D(X) \geq w, D(Y)<y, D(Z) \geq y$, robot $Y$ seeks coalition, but robots $X$ and $Z$ do not, so based on Theorem 2 we define

$$
P_{o, 7}(X)=P_{o, 6}(X) .
$$

Case 8: When $D(X) \geq w, D(Y) \geq y, D(Z) \geq y$, all of the robots do not seek coalition, so based on Theorem 2 we define

$$
P_{o, 8}(X)=P_{o, 6}(X) .
$$

We define some notations as follows:

$$
\begin{aligned}
\Omega_{i}(w, y) & =\{(D(X), D(Y), D(Z)) \mid \text { Case } i\} \\
\chi & \triangleq(D(X), D(Y), D(Z)) \\
d \chi & \triangleq d D(X) d D(Y) d D(Z) .
\end{aligned}
$$

Let $f(w, y)$ denote the average payoff of the $w$ strategist in a population of $y$ strategists. Then, we have the following theorem.
Theorem 5: The average payoff for a mutant with strategy $w$ when the population has strategy $y$ (i.e., Problem 1) is given as

$f(w, y)$

$=\sum_{i=1}^{8}\left\{\iiint_{\chi \in \Omega_{i}(w, y)} P_{o, i}(X) g(D(X)) g(D(Y)) g(D(Z)) d \chi\right\}$.

Further, we can simplify it as

$$
\begin{aligned}
f(w, y)=\frac{1}{L^{3}}[ & \frac{2 L^{4}+y^{3} w+w^{2} y^{2}-w^{2} y L+3 y^{2} w L}{36 L} \\
& +\frac{V\left(2 L^{3}+w y^{2}-w y L\right)}{18 L \mu}-\theta w L^{2} \\
& -\frac{V}{9 L \mu} e^{-\frac{3 \mu L}{V}} \frac{V^{3}}{\mu^{3}}\left(e^{\frac{\mu}{V} w}-1\right)\left(e^{\frac{\mu}{V} y}-1\right)^{2} \\
& -\frac{V}{6 L \mu} e^{-\frac{3 \mu L}{2 V}} \frac{(2 V)^{3}}{\mu^{3}}\left(e^{\frac{\mu}{2 V} w}-1\right) \\
& \times\left(e^{\frac{\mu}{2 V} y}-1\right)\left(e^{\frac{\mu}{2 V} L}-e^{\frac{\mu}{2 V} y}\right) \\
& -\frac{V}{9 L \mu} e^{-\frac{3 \mu L}{2 V}} \frac{(2 V)^{3}}{\mu^{3}}\left(e^{\frac{\mu}{2 V} L}-e^{\frac{\mu}{2 V} w}\right) \\
& \times\left(e^{\frac{\mu}{2 V} y}-1\right)^{2}-\frac{V}{9 L \mu} e^{-\frac{\mu L}{V}} \frac{(3 V)^{3}}{\mu^{3}} \\
& \times\left(e^{\frac{\mu}{3 V} w}-1\right)\left(e^{\frac{\mu}{3 V} L}-e^{\frac{\mu}{3 V} y}\right)^{2} \\
& -\frac{V}{9 L \mu} e^{-\frac{\mu L}{V}} \frac{(3 V)^{3}}{\mu^{3}}\left(e^{\frac{\mu}{3 V} L}-e^{\frac{\mu}{3 V} w}\right) \\
& \left.\times\left(e^{\frac{\mu}{3 V} L}+e^{\frac{\mu}{3 V} y}-2\right)\left(e^{\frac{\mu}{3 V} L}-e^{\frac{\mu}{3 V} y}\right)\right] .
\end{aligned}
$$

Proof:

$$
\begin{array}{r}
f(w, y)=\sum_{i=1}^{8}\left\{\int_{\chi \in \Omega_{i}(w, y)} P_{o, i}(X) g(D(X))\right. \\
\times g(D(Y)) g(D(Z)) d \chi\} \\
=\frac{1}{L^{3}} \sum_{i=1}^{8}\left\{\iiint_{\chi \in \Omega_{i}(w, y)} P_{o, i}(X) d \chi\right\} .
\end{array}
$$

By symmetry, we have

$$
\iiint_{\chi \in \Omega_{2}(w, y)} P_{o, 2}(X) d \chi=\iiint_{\chi \in \Omega_{3}(w, y)} P_{o, 3}(X) d \chi .
$$

By deriving each integration of $P_{o, i}(X)$ (detail are omitted to the limited space), we can obtain (15). 


\section{B. Analysis of ESS}

Theorem 6: $y=0$ is an ESS. In other words, separately detecting in a triad of robots without coalition always pays.

Proof: From (10) and (11), we can easily know that to each $w, 0<w \leq L$, we have

$$
\begin{aligned}
& f(w, 0) \\
& =\frac{1}{L^{3}} \iiint_{\substack{D(X) \geq w, D(Y) \geq 0, D(Z) \geq 0}}\left(\frac{D(X)}{9 L}+\frac{V}{9 L \mu}-\frac{V}{9 L \mu} e^{-\frac{\mu Q}{3 V}}\right) d \chi
\end{aligned}
$$

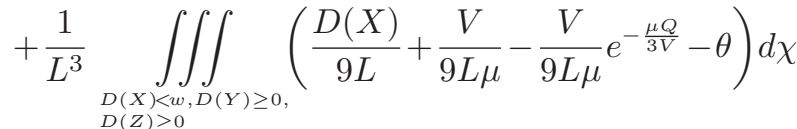

$f(0,0)$

$$
\begin{gathered}
=\frac{1}{L^{3}} \quad \iiint_{\substack{D(X) \geq 0, D(Y) \geq 0, D(Z) \geq 0}}\left(\frac{D(X)}{9 L}+\frac{V}{9 L \mu}-\frac{V}{9 L \mu} e^{-\frac{\mu Q}{3 V}}\right) d \chi \\
=\frac{1}{L^{3}} \quad \iiint_{\substack{D(X) \geq w, D(Y) \geq 0, D(Z) \geq 0}}\left(\frac{D(X)}{9 L}+\frac{V}{9 L \mu}-\frac{V}{9 L \mu} e^{-\frac{\mu Q}{3 V}}\right) d \chi \\
+\frac{1}{L^{3}} \quad \iiint_{\substack{D(X)<w, D(Y) \geq 0, D(Z) \geq 0}}\left(\frac{D(X)}{9 L}+\frac{V}{9 L \mu}-\frac{V}{9 L \mu} e^{-\frac{\mu Q}{3 V}}\right) d \chi .
\end{gathered}
$$

Obviously, we have $f(w, 0)<f(0,0)$ when $0<w \leq L$ and $0<\theta<+\infty$. Therefore, we can draw a conclusion that 0 is always an ESS.

Theorem 7: 1) $y=L$ is an ESS when $\theta \in\left(0, \theta_{0}\right]$, where

$$
\theta_{0}=1 / 9+f_{0} L
$$

and 2) $y=L$ is an ESS when $\theta \in\left(0, \theta_{1}\right)$, and $L$ is not an ESS when $\theta \in\left(\theta_{1},+\infty\right)$, where $\theta_{1} \geq \theta_{0}$, and

$$
\begin{aligned}
\theta_{1} & =\min \left(\theta_{2}, \theta_{3}, \theta_{4}\right) \\
c & =\frac{V}{9 L \mu} e^{-\frac{3 \mu L}{V}}\left(\frac{V^{2}}{\mu^{2}}\right) \frac{1}{L^{3}}\left(e^{\frac{\mu L}{V}}-1\right)^{2} \\
d & =\frac{V}{9 L \mu} e^{-\frac{3 \mu L}{2 V}}\left(\frac{4 V^{2}}{\mu^{2}}\right) \frac{1}{L^{3}}\left(e^{\frac{\mu L}{2 V}}-1\right)^{2} \\
\theta_{2} & =1 / 9+L d^{2} /(4 c) \\
\theta_{3} & \triangleq \frac{1}{9}-c\left(e^{\frac{\mu L}{V}}-1\right) \frac{V}{\mu}+d\left(e^{\frac{\mu L}{2 V}}-1\right) \frac{2 V}{\mu} \\
\theta_{4} & =\frac{1}{9}-c L e^{\frac{\mu L}{V}}+d L e^{\frac{\mu L}{2 V}} \\
f_{0} & \triangleq \min \left\{-c+d,-c e^{\frac{\mu L}{V}}+d e^{\frac{\mu L}{2 V}}\right\} \\
& =\min _{0 \leq w \leq L}\left\{-c e^{\frac{\mu w}{V}}+d e^{\frac{\mu w}{2 V}}\right\} .
\end{aligned}
$$

In other words, always forming a three-way coalition (i.e., always detecting all together) pays when the cost is below a threshold.
Proof: From (14), (6), and (9), we know

$$
\begin{aligned}
f(w, L)= & \iiint_{\substack{0<D(X) \leq w \\
0<D(Y) \leq L \\
0<D(Z) \leq L}} \frac{1}{L^{3}}\left(\frac{D(X)+D(Y)+D(Z)}{9 L}\right. \\
& \left.+\int \frac{V}{9 L \mu}-\frac{V}{9 L \mu} e^{\frac{-\mu Q}{V}}-\theta\right) d \chi \\
& \quad \iint_{\substack{w \leq D(X) \leq L \\
0<D(Y) \leq L \\
0<D(Z) \leq L}} \frac{1}{L^{3}}\left(\frac{D(X)}{9 L}+\frac{V}{9 L \mu}-\frac{V}{9 L \mu} e^{\frac{-\mu\left(\frac{Q}{2}\right)}{V}}\right) d \chi \\
= & \frac{1}{18}+\frac{w}{9 L}+\frac{V}{9 L \mu}-\frac{w \theta}{L}-\frac{V c e^{\frac{\mu w}{V}}}{\mu} \\
& +\frac{V c}{\mu}-\frac{2 V d e^{\frac{\mu L}{2 V}}}{\mu}+\frac{2 V d e^{\frac{\mu w}{2 V}}}{\mu} .
\end{aligned}
$$

Note that in the preceding integration $Q$ is not constant. Let $m(w)=e^{\mu w /(2 V)}$, and then we have

$$
\frac{\partial f(w, L)}{\partial w}=\frac{1}{9 L}-\frac{\theta}{L}-c(m(w))^{2}+d m(w) .
$$

Since $-c e^{\mu w / V}+d e^{\mu w / 2 V}=-c(m(w))^{2}+d m(w)$, and $\partial^{2}\left(-c(m(w))^{2}+d m(w)\right) / \partial^{2} m(w)<0$ (a quadratic function), we define $f_{0}$ in (23), and we have

$$
\begin{aligned}
\frac{d}{c} & =\frac{4 e^{\frac{3 \mu L}{2 V}}}{\left(e^{\frac{\mu L}{2 V}}+1\right)^{2}}>\frac{4 e^{\frac{\mu L}{V}}}{\left(e^{\frac{\mu L}{2 V}}+e^{\frac{\mu L}{2 V}}\right)^{2}}=1 \\
\frac{d e^{\frac{\mu L}{2 V}}}{c e^{\frac{\mu L}{V}}} & =\frac{4 e^{\frac{\mu L}{V}}}{\left(e^{\frac{\mu L}{2 V}}+1\right)^{2}}>\frac{4 e^{\frac{\mu L}{V}}}{\left(e^{\frac{\mu L}{2 V}}+e^{\frac{\mu L}{2 V}}\right)^{2}}=1 .
\end{aligned}
$$

Therefore, we have $-c+d>0$, and $-c e^{\mu L / V}+d e^{\mu L /(2 V)}>$ 0 . Therefore, we know $f_{0}>0$. If $1 /(9 L)-\theta / L+f_{0} \geq 0$, i e., $\theta \leq \theta_{0}=1 / 9+f_{0} L$, then we can obtain

$$
\begin{aligned}
\frac{\partial f(w, L)}{\partial w} & >\frac{1}{9 L}-\frac{\theta}{L}+f_{0}>0, \text { when } 0<w<L \\
f(L, L) & >f(w, L), \text { to any } w, 0<w<L .
\end{aligned}
$$

From (26), we can know that $L$ is an ESS (strategy $L$ means always trying to make a coalition with other robots) when $\theta \leq \theta_{0}$. This just approved the first part of Theorem 7 .

The expression (16) has supplied a value range for $\theta$, which could lead to an ESS $L$. However, beyond this value range, there may be other values for $\theta$ that could also lead to an ESS $L$. Therefore, in the following derivation, we will try to find out all of those values as well. We know that $\partial f(w, L) / \partial w$ is a quadratic function of $m$ with a factor $-c$ of $(m(w))^{2}$. We now let $\partial f(w, L) / \partial w=0$ and find the solutions. We know that there are two possible solutions that we denote as $m_{0}$ and $m_{1}$, and $m_{0}<m_{1}$, i.e.,

$$
\begin{aligned}
\delta & =d^{2}-4(-c(1 /(9 L)-\theta / L)) \\
m_{0} & =(-d+\sqrt{\delta}) /(-2 c) \\
m_{1} & =(-d-\sqrt{\delta}) /(-2 c) .
\end{aligned}
$$

Let us first consider the term $\delta$. By recalling the shape of a quadratic function with a negative factor in the fist term, we can 
easily obtain that if $\delta \leq 0, \partial f(w, L) / \partial w<0$ to every value of $w, 0 \leq w \leq L$, except for at most one point $w_{0}$, which satisfies $\partial f\left(w_{0}, L\right) / \partial w=0$. Therefore, we know that when $\delta \leq 0, L$ is not an ESS. That $L$ is an ESS leads to $\delta>0$, namely, that $\theta<\theta_{2}$, where $\theta_{2}$ is defined in (20).

From (24), (27)-(29), and (20), considering the shape of the quadratic function as (24), we can know that Lemma 1 is true. Then, we define $\theta_{1}=\min \left(\theta_{2}, \theta_{3}, \theta_{4}\right)$, which is the same as in (17). We can obtain the following:

1) When $\theta \in\left(0, \theta_{1}\right), L$ is an ESS.

2) When $\theta \in\left(\theta_{1},+\infty\right)$, at least one of $\theta \leq \theta_{2}, \theta \leq \theta_{3}$, and $\theta \leq \theta_{4}$ does not hold, and therefore $L$ is not an ESS.

3) When $\theta=\theta_{1}$, whether $L$ is an ESS can be concluded by some more complicated approaches. However, since we have made the conclusion whether $L$ is an ESS versus $\theta$ varying from 0 to infinity except a point $\theta_{1}$, this point $\theta_{1}$ seems somewhat trivial for our discussion. We can just ignore it.

Therefore, we conclude the proof of Theorem 7.

Let $h(w, y, z)$ denote the average payoff that three individuals play strategies $w, y$, and $z$, respectively. We have $f(w, y)=$ $h(w, y, y)$. Then, we have the following lemma.

Lemma 1: If $\theta<\theta_{2}$ and $m(L)=e^{\mu L /(2 V)}$, then $L$ is an ESS if and only if condition (a) is satisfied, and either condition (b) or both conditions (c) and (d) are satisfied.

a) $m_{0}<m(L) \leq m_{1}$, i.e., $d-2 c m(L)<\sqrt{\delta}$ and $2 c m(L)-$ $d \leq \sqrt{\delta}$ (i.e., when $2 \mathrm{~cm}(L)-d>0, \theta \leq \theta_{4}$; and when $\left.2 c m(L)-d \leq 0, \theta<\theta_{4}\right)$.

b) $f(L, L)>f(0, L)$, i.e., $\theta<\theta_{3}$.

c) $f(L, L)=f(0, L)$, i.e., $\theta=\theta_{3}$.

d) For all $w, 0 \leq w<L, h(L, 0, L)>h(w, 0, L)$.

Proof: First we prove some equivalency.

1) $m_{0}<m(L) \leq m_{1} \Leftrightarrow(-d+\sqrt{\delta}) /(-2 c)<m(L) \leq$ $(-d-\sqrt{\delta}) /(-2 c) \Leftrightarrow d-2 c m(L)<\sqrt{\delta}$ and $2 c m(L)-$ $d \leq \sqrt{\delta} \Leftrightarrow$ when $2 c m(L)-d>0, \quad 2 c m(L)-d \leq$ $\sqrt{d^{2}-4(-c(1 /(9 L)-\theta / L))} ;$ and when $2 c m(L)-$ $\left.d \leq 0, \quad d-2 c m(L)<\sqrt{d^{2}-4(-c(1 /(9 L)-\theta / L))}\right) ;$ $\Leftrightarrow$ when $2 c m(L)-d>0, \theta \leq \theta_{4}$; and when $2 c m(L)-$ $d \leq 0, \theta<\theta_{4}$.

2)

$$
\begin{aligned}
& f(L, L)>f(0, L) \\
& \Leftrightarrow f(L, L)=\frac{3}{18}+\frac{V}{9 L \mu}-\frac{L \theta}{L}-\frac{V c e^{\frac{\mu L}{V}}}{\mu}+\frac{V c}{\mu}> \\
& \quad f(0, L)=\frac{1}{18}+\frac{V}{9 L \mu}-\frac{2 V d e^{\frac{\mu L}{2 V}}}{\mu}+\frac{2 V d}{\mu} \\
& \Leftrightarrow \theta<\frac{1}{9}-c\left((m(L))^{2}-1\right) \frac{V}{\mu}+d(m(L)-1) \frac{2 V}{\mu}=\theta_{3} .
\end{aligned}
$$

3) $f(L, L)=f(0, L) \Leftrightarrow \theta=\theta_{3}$.

4) $\theta<\theta_{2} \Leftrightarrow \delta>0$.

Next, we will prove the "if and only if" part in the theorem:

1) To Prove $\Rightarrow$ : In the proof of Theorem 7, we have proved that $L$ is an ESS $\Rightarrow \delta>0$.

From the definition of an ESS, we can easily know that $L$ is an ESS $\Rightarrow$ when either condition (b) or both conditions (c) and (d) are correct.
Further, we will prove by contradiction that $m(L)>m_{1}$ or $m(L) \leq m_{0} \Rightarrow L$ is not an ESS. If $m(L)>m_{1}$, then we know that $\partial f(L, L) / \partial w<0$. Since $\partial f(w, L) / \partial w$ is a continuous function, we know that there exists $w_{1}, 0<w_{1}<L$, and that $\partial f(w, L) / \partial w<0$ when $w_{1} \leq w<L$. Therefore, $f\left(w_{1}, L\right)>$ $f(L, L)$, and from this, we can say that $L$ is not an ESS. This is a contradiction.

If $m(L) \leq m_{0}$, since $\partial f(w, L) / \partial w$ is a quadratic function of $m(w)$, then we know that there exists $w_{2}, 0<w_{2}<L$, and that $\partial f(w, L) / \partial w<0$ when $w_{2} \leq w<L$. Therefore, $f\left(w_{2}, L\right)>$ $f(L, L)$, and from this, we can say that $L$ is not an ESS. This is a contradiction.

2) To Prove $\Leftarrow$ : When $\delta>0, m_{0}$ and $m_{1}$ are real numbers. From condition (a), i.e., $m_{0}<m(L) \leq m_{1}$, and the fact that $m(w)$ is an increasing faction of $w$, we know that either $f(w, L)$ is a monotonically increasing function of $w$ when $0 \leq w \leq L$, or that $f(w, L)$ is a function of $w$ that is first a monotonically decreasing and then monotonically increasing function when $0 \leq w \leq L$. Therefore, there are at least one and at most two maximum points for the function $f(w, L)$. Furthermore, the maximum point(s) must be 0 or $L$.

Since condition (b) or both conditions (c) and (d) are satisfied, we have either $f(L, L)>f(0, L)$ or $f(L, L)=f(0, L)$.

If $f(L, L)>f(0, L)$, then there are only one maximum point for $f(w, L)$, which is $L$. Then, $L$ is an ESS.

If $f(L, L)=f(0, L)$, then there are two maximum points for $f(w, L)$. From the definition of an ESS, if for each $w \in$ $[0, L), h(L, 0, L)>h(w, 0, L)$, then $L$ is an ESS.

Therefore, we have partially solved Problem 2. Finally, ESS studies for strategy $y, 0<y<L$, will be included in our future work [49] and its corresponding journal version paper, in which, we will show that a true coalition is likely to arise only in the case that an interior ESS exists.

\section{EVALUATION}

In this section, we provide some evaluations under the parameters $L=10$ unit, $\mu=1$ unit, and $V=1$ unit.

\section{A. Payoff of the Potential Mutant}

In Fig. 2, we let the population adopt a strategy $y=0$, fix $\theta=0,0.25,0.5,0.75$, and 1 , and let $w$ increase from 0 to $L$ with an increment of $0.1 \mathrm{~L}$. As illustrated in Fig. 2, with each fixed $\theta$, the payoff for the potential mutant decreases when $w$ increases. This confirms our conclusion in the last section that 0 is always an ESS. Fig. 2 also shows that, with any fixed $w$, when $\theta$ increases, the payoff for the potential mutant decreases. We can then see that 0 is always an ESS. It makes sense because the potential mutant will never benefit but only pay the investment cost to try to form a coalition when the strategy of the others (population) is not to try to form a coalition.

In Figs. 3 and 4, we let the population adopt a strategy $y=L$. We can then obtain $\theta_{1}$ defined before. In Fig. 3, we fix $\theta=0$, $0.25 \theta_{1}, 0.5 \theta_{1}, 0.75 \theta_{1}$, and $1 \theta_{1}$, and let $w$ increase from 0 to $L$ with an increment of $0.1 L$. As illustrated in Fig. 3, with each fixed $\theta$, the payoff for the potential mutant increases when $w$ increases. This confirms our conclusion in the last section that 


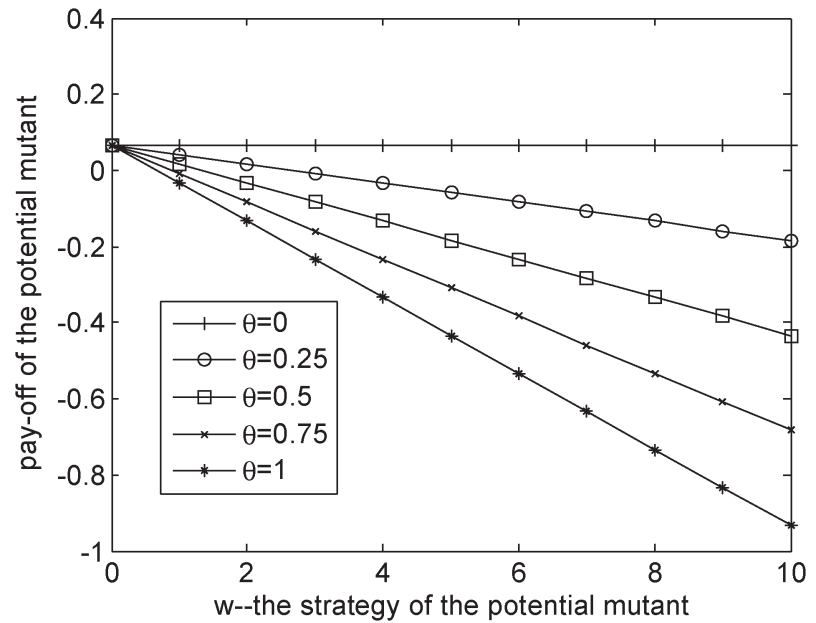

Fig. 2. Payoff of the potential mutant versus $w$, with the population adopting a strategy $y=0$.

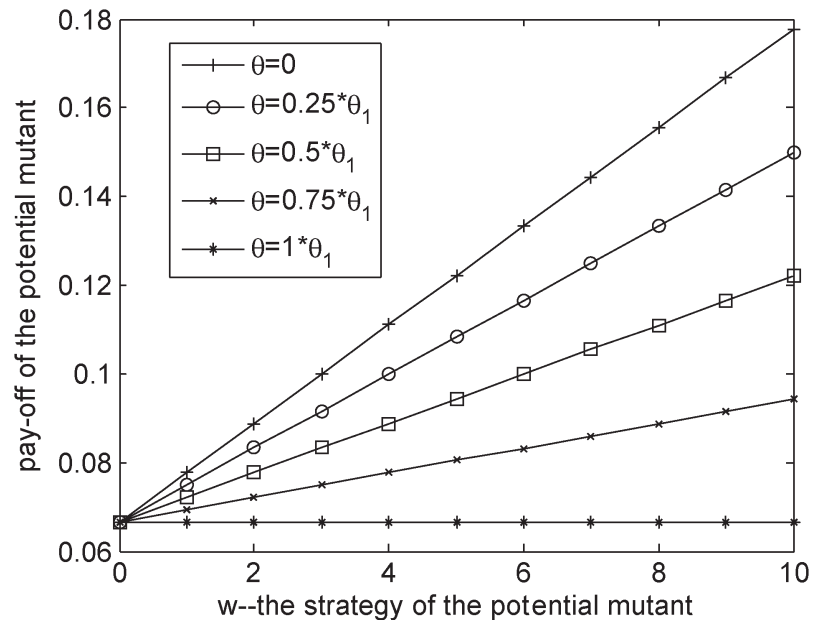

Fig. 3. Payoff of the potential mutant versus $w$, with the population opting a strategy $y=L$, and $\theta \leq \theta_{1}$.

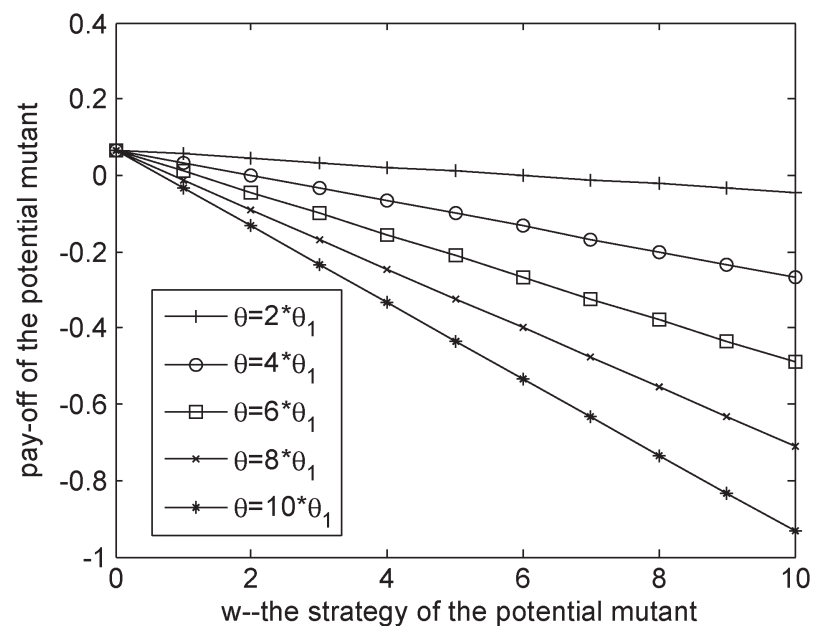

Fig. 4. Payoff of the potential mutant versus $w$, with the population opting a strategy $y=L$ and $\theta>\theta_{1}$.

$L$ is an ESS when $\theta<\theta_{1}$. In addition, from Fig. 3, with any fixed $w$, when $\theta$ increases, the payoff for the potential mutant decreases. In Fig. 3, we observe that the investment cost is below a threshold, always to try to form a coalition is an ESS.
This is because when the investment cost is small, the potential mutant can still benefit most by forming a three-way coalition with others with strategy $L$.

In Fig. 4, we fix $\theta=2 \theta_{1}, 4 \theta_{1}, 6 \theta_{1}, 8 \theta_{1}$, and $10 \theta_{1}$, and let $w$ increase from 0 to $L$ with an increment of $0.1 L$. As illustrated in Fig. 4, with each fixed $\theta$, the payoff for the potential mutant decreases when $w$ increases. This confirms our conclusion in the last section that $L$ is not an ESS when $\theta>\theta_{1}$. In addition, from Fig. 4, with any fixed $w$, when $\theta$ increases, the payoff for the potential mutant decreases. In Fig. 4, we observe that, when the investment cost is above a threshold, always trying to form a coalition is not an ESS. This is because the investment cost is large enough to tradeoff the benefit for the potential mutant that forms a three-way coalition with others with strategy $L$.

\section{CONClusion AND Future Work}

The purpose of this paper has been to study collaborative robots via mathematical modeling. The modeling provides us with deep insights into the impacts of collaborations and can provide guides on how to collaborate in collaboration systems and to understand the tradeoff of collaboration and cost.

Inspired by the society of animals, we have studied coalition formation of three robots for detecting intrusion objects over a closed border curve. We have derived expressions for the payoff for the mutant robot in eight cases, and also the expression of the average payoff for the mutant robot.

As to the discussion in ESS, we have obtained a result that 0 (equivalent to never trying forming a coalition) is always an ESS, and that when $\theta$ is below a threshold $\theta_{1}, L$ (equivalent to always trying forming a coalition) is an ESS.

Our future work is to derive some results of the formation of the true coalition [49]. We will try to find out the condition under which a strategy between 0 and $L$ is an ESS. That condition is also the condition under which the formation of a true coalition is possible.

\section{REFERENCES}

[1] S. A. Pandit and C. P. van Schaik, "A model for leveling coalitions among primate males: Toward a theory of egalitarianism," Behav. Ecol. Sociobiol., vol. 55, pp. 161-168, 2003.

[2] R. Noë, "A model of coalition formation among male baboons with fighting ability as the crucial parameter," Anim. Behav., vol. 47, no. 1, pp. 211-213, Jan. 1994.

[3] R. Noë and A. A. Sluijter, "Which adult male savanna baboons form coalitions," Int. J. Primatol., vol. 16, no. 2, pp. 77-105, Apr. 1995.

[4] L. A. Dugatkin, "A model of coalition formation in animals," Proc. $R$. Soc. Lond., B Biol. Sci., vol. 265, no. 1410, pp. 2121-2125, Nov. 1998.

[5] C. Packer, D. A. Gilbert, A. E. Pusey, and S. J. O'Brien, "A molecular genetic analysis of kinship and co-operation in African lions," Nature, vol. 351, no. 6327, pp. 562-565, Jun. 1991.

[6] R. Noë, "A veto game played by baboons: A challenge to the use of the Prisoner's Dilemma as a paradigm for reciprocity and cooperation," Anim. Behav., vol. 39, no. 1, pp. 78-90, Jan. 1990.

[7] H. Whitehead and R. Connor, "Alliances II. How large should alliances be?" Anim. Behav., vol. 69, pp. 117-126, 2005.

[8] R. Connor and H. Whitehead, "Alliances II. Rates of encounter during resource utilization: A general model of intrasexual alliance formation in fission-fusion societies," Anim. Behav, vol. 69, no. 1, pp. 127-132, Jan. 2005.

[9] R. Noë, "Alliance formation among male baboons: Shopping for profitable partners," in Coalitions and Alliances in Humans and Other Animals, A. H. Harcourt and F. B. M. de Waal, Eds. Oxford, U.K.: Oxford Univ. Press, 1992, pp. 285-321.

[10] M. Mesterton-Gibbons, An Introduction to Game-Theoretic Modeling. Providence, RI: AMS, 2001. 
[11] T. Caro, Antipredator Defenses in Birds and Mammals. Chicago, IL: Univ. Chicago Press, 2005.

[12] L. A. Dugatkin, "Breaking up fights between others: A model of intervention behaviour," Proc. R. Soc. Lond., B Biol. Sci., vol. 265, no. 1394, pp. 433-436, Mar. 1998.

[13] T. M. Caro, "Cheetahs of the Serengeti Plains: Group living in an asocial species," in Wildlife Behaviour and Ecology. Chicago, IL: Univ. Chicago Press, 1994.

[14] F. B. M. de Waal and A. H. Harcourt, "Coalitions and alliances: A history of ethological research," in Coalitions and Alliances in Humans and Other Animals, A. H. Harcourt and F. B. M. de Waal, Eds. Oxford, U.K.: Oxford Univ. Press, 1992, pp. 1-19.

[15] F. B. Bercovitch, "Coalitions, cooperation, and reproductive tactics among adult male baboons," Anim. Behav., vol. 36, pp. 1198-1209, 1988

[16] M. Mesterton-Gibbons and T. N. Sherratt, "Coalition formation: A gametheoretic analysis," Behav. Ecol., vol. 18, no. 2, pp. 277-286, Mar. 2007.

[17] C. J. Zabel, S. E. Glickman, L. G. Frank, K. B. Woodmansee, and G. Keppel, "Coalition formation in a colony of prepubertal spotted hyaenas," in Coalitions and Alliances in Humans and Other Animals, A. H. Harcourt and F. B. M. de Waal, Eds. Oxford, U.K.: Oxford Univ. Press, 1992, pp. 113-135.

[18] R. A. Johnstone and L. A. Dugatkin, "Coalition formation in animals and the nature of winner and loser effects," Proc. R. Soc. Lond., B Biol. Sci., vol. 267, no. 1438, pp. 17-21, Jan. 2000.

[19] R. C. Connor, R. A. Smolker, and A. F. Richards, "Dolphin alliances and coalitions," in Coalitions and Alliances in Humans and Other Animals, A. H. Harcourt and F. B. M. de Waal, Eds. Oxford, U.K.: Oxford Univ. Press, 1992, pp. 415-443.

[20] J. M. Smith, Evolution and the Theory of Games. Cambridge, U.K.: Cambridge Univ. Press, 1982

[21] M. Enquist and O. Leimar, "Evolution of fighting behaviour: Decision rules and assessment of relative strength," J. Theor. Biol., vol. 102, pp. $387-410,1983$.

[22] I. Eshel, "Evolutionary and continuous stability," J. Theor. Biol., vol. 103, pp. 99-111, 1983

[23] [Online]. Available: http://en.wikipedia.org/wiki/Evolutionary_stable_ strategy

[24] L. J. Morrell, P. R. Y. Backwell, and N. B. Metcalfe, "Fighting in fiddler crabs Uca mjoebergi: What determines duration?" Anim. Behav., vol. 70, no. 3, pp. 653-662, Sep. 2005.

[25] L. A. Dugatkin, "Game theory and cooperation," in Game Theory and Animal Behavior, L. A. Dugatkin and H. K. Reeve, Eds. New York: Oxford Univ. Press, 1998, pp. 38-63.

[26] C. Boehm, Hierarchy in the Forest: The Evolution of Egalitarian Behavior. Cambridge, MA: Harvard Univ. Press, 1999.

[27] J. N. McDonald, North American Bison. Berkeley, CA: Univ. California Press, 1981

[28] F. B. Christiansen, "On conditions for evolutionary stability for a continuously varying character," Am. Nat., vol. 138, pp. 37-50, 1991.

[29] A. L. Engh, E. R. Siebert, D. A. Greenberg, and K. E. Holekamp, "Patterns of alliance formation and postconflict aggression indicate spotted hyaenas recognize third-party relationships," Anim. Behav., vol. 69, no. 1, pp. 209217, Jan. 2005.

[30] J. B. Silk, S. C. Alberts, and J. Altmann, "Patterns of coalition formation by adult female baboons in Amboseli, Kenya," Anim. Behav., vol. 67, no. 3, pp. 573-582, Mar. 2004.

[31] O. Kempthorne and L. Folks, Probability, Statistics, and Data Analysis. Ames, IA: Iowa State Univ. Press, 1971.

[32] C. Packer, "Reciprocal altruism in Papio anubis," Nature, vol. 265, no. 5593 , pp. $441-443,1977$.

[33] R. C. Connor, R. M. Heithaus, and L. M. Barre, "Superalliance of bottlenose dolphins," Nature, vol. 397, no. 6720, pp. 571-572, Feb. 1999.

[34] C. K. Hemelrijk, "Support for being groomed in long-tailed macaques, Macaca fascicularis," Anim. Behav., vol. 48, no. 2, pp. 479-481, Aug. 1994.

[35] J. Moya-Larano and D. H. Wise, "Survival regression analysis: A powerful tool for evaluating fighting and assessment," Anim. Behav., vol. 60, no. 3, pp. 307-313, Sep. 2000.

[36] J. M. Smith and G. R. Price, "The logic of animal conflict," Nature, vol. 246, no. 5427, pp. 15-18, Nov. 1973.

[37] L. Barrett and S. P. Henzi, "The utility of grooming in baboon troops," in Economics in Nature: Social Dilemmas, Mate Choice and Biological Markets, R. Noë, J. A. R. A. M. van Hooff, and P. Hammerstein, Eds. Cambridge, U.K.: Cambridge Univ. Press, 2001.

[38] Y. Hsu and L. L. Wolf, "The winner and loser effect: Integrating multiple experiences," Anim. Behav., vol. 57, no. 4, pp. 903-910, Apr. 1999.

[39] M. Bulmer, Theoretical Evolutionary Ecology. Sunderland, MA: Sinauer, 1994
[40] C. Rutte, M. Taborsky, and M. W. G. Brinkhof, "What sets the odds of winning and losing?" Trends Ecol. Evol., vol. 21, no. 1, pp. 16-21, Jan. 2006.

[41] X. Liang and Y. Xiao, "Modeling collaborative robots for detecting intrusions," in Proc. Int. Symp. CTS, 2009, pp. 306-313.

[42] Y. Xiao and Y. Zhang, "Surveillance and tracking system with collaboration of robots, sensor nodes, and RFID tags," in Proc. SN 18th Int. Conf., ICCN 2009, San Francisco, CA, Aug. 2009, pp. 1-6.

[43] Y. Xiao and X. Liang, "Bio-inspired communications among robots, sensors, and RFID tags," in Proc. ICMA , 2009, pp. 3573-3578.

[44] Y. Zhang and Y. Xiao, "Primate-inspired scent marking for mobile and static sensors and RFID tags," in Proc. SN 18th Int. Conf., ICCN 2009, San Francisco, CA, Aug. 2009, pp. 1-5.

[45] Y. Zhang and Y. Xiao, "A boundary surveillance strategy based on critical line in wireless sensor networks," in Proc. ICMA, 2009, pp. 459-464.

[46] Y. Zhang, Y. Xiao, and K. L. Bales, "Primate social systems, scentmarking, and their applications in mobile and static sensor networks," Int. J. Sensor Netw., vol. 5, no. 4, pp. 210-222, Aug. 2009.

[47] J. Liu, Y. Xiao, Q. Hao, and K. Ghaboosi, "Bio-inspired visual attention in agile sensing for target detection," Int. J. Sensor Netw., vol. 5, no. 2, pp. 98-111, Apr. 2009

[48] Y. Xiao and Y. Zhang, "Divide and conquer based surveillance framework using robots, sensor nodes, and RFID tags," Wireless Commun. Mobile Comput. (WCMC), DOI: 10.1002/wcm.863. [Online]. Available: http://www3.interscience.wiley.com/journal/122667039/abstract

[49] X. Liang and Y. Xiao, "Bio-inspired true coalition formation on intrusion detection by mobile robots," in Proc. CollaborateCom, 2009.

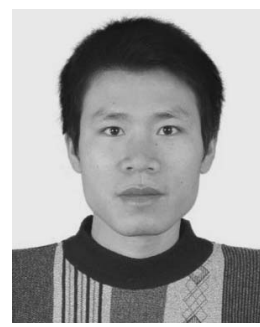

Xiannuan Liang received the B.S. and M.S. degrees from Jilin University, Changchun, China, in 2004 and 2007, respectively, both in mathematics. $\mathrm{He}$ is currently a graduate student with the Department of Computer Science, University of Alabama, Tuscaloosa.

Under the supervision of Prof. Y. Xiao with the Department of Computer Science, University of Alabama, his current research areas are sensor networks and wireless networks.

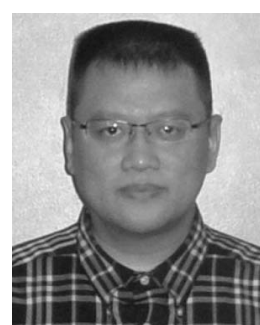

Yang Xiao (SM'04) received the B.S. and M.S degrees from Jilin University, Changchun, China, and the M.S. and Ph.D. degrees in computer science and engineering from Wright State University, Dayton, $\mathrm{OH}$.

$\mathrm{He}$ worked in the industry as a Medium Access Control (MAC) Architect involved in the IEEE 802.11 standard enhancement work before he joined the academia. He is currently with the Department of Computer Science (with tenure), The University of Alabama, Tuscaloosa. He is also a Guest Professor with Jilin University from 2007 to 2012 and an Adjunct Professor with Zhejiang University, Hangzhou, China, from 2007 to 2009. He currently serves as the Editor-in-Chief for the International Journal of Security and Networks, the International Journal of Sensor Networks, and the International Journal of Telemedicine and Applications. His research has been supported by the US National Science Foundation (NSF), U.S. Army Research, Fleet \& Industrial Supply Center San Diego (FISCSD), and The University of Alabama's Research Grants Committee. He has published more than 300 papers in major journals, refereed conference proceedings, and book chapters. His research areas are security, telemedicine, robot, sensor networks, and wireless networks.

Dr. Xiao was a voting member of IEEE 802.11 Working Group from 2001 to 2004. He is a member of American Telemedicine Association. He serves as a panelist for the U.S. NSF, Canada Foundation for Innovation (CFI)'s Telecommunications Expert Committee, and the American Institute of Biological Sciences (AIBS), as well as a referee/reviewer for many national and international funding agencies. He serves on the technical program committee for more than 100 conferences such as the IEEE Conference on Computer Communications (INFOCOM), the International Conference on Distributed Computing Systems (ICDCS), the International Symposium on Mobile Ad Hoc Networking and Computing (MOBIHOC), the International Conference on Communications (ICC), the IEEE Global Telecommunications Conference (GLOBECOM), and the IEEE Wireless Communication Networking Conference (WCNC). He serves as an Associate Editor for several journals, e.g., IEEE TRANSACTIONS ON VEHICULAR TECHNOLOGY. 